\title{
Application of endobronchial ultrasonography using a guide sheath and electromagnetic navigation bronchoscopy in the diagnosis of atypical bacteriologically-negative pulmonary tuberculosis
}

\author{
Ye Gu${ }^{1}$, Chunyan $\mathrm{Wu}^{2}$, Fangyou Yu ${ }^{3}$, Xuwei Gui ${ }^{4}$ Jun $\mathrm{Ma}^{4}$, Liping Cheng ${ }^{4}$, Qin Sun ${ }^{4}$, Wei Sha ${ }^{4}$ \\ ${ }^{1}$ Department of Bronchoscopy, ${ }^{2}$ Department of Pathology, ${ }^{3}$ Clinical Laboratory, Shanghai Pulmonary Hospital, Tongji University School of \\ Medicine, Shanghai 200433, China; ${ }^{4}$ Clinic and Research Center of Tuberculosis, Shanghai Key Laboratory of Tuberculosis, Shanghai Pulmonary \\ Hospital, Tongji University School of Medicine, Shanghai 200433, China \\ Contributions: (I) Conception and design: Y Gu; (II) Administrative support: W Sha; (III) Provision of study materials or patients: Y Gu, C Wu, \\ F Yu, X Gui, J Ma, L Cheng, Q Sun; (IV) Collection and assembly of data: Y Gu, C Wu, F Yu, X Gui, J Ma, L Cheng, Q Sun; (V) Data analysis and \\ interpretation: Y Gu; (VI) Manuscript writing: All authors; (VII) Final approval of manuscript: All authors. \\ Correspondence to: Wei Sha. Department of Tuberculosis, Shanghai Pulmonary Hospital, Tongji University School of Medicine, 507 Zheng Min Road, \\ Shanghai 200433, China. Email: shfksw@126.com; Qin Sun. Department of Tuberculosis, Shanghai Pulmonary Hospital, Tongji University School \\ of Medicine, 507 Zheng Min Road, Shanghai 200433, China. Email: sunqinbonjour@163.com.
}

Background: In the absence of a positive sputum bacteriological result, pathological and bacteriological examinations of lung lesion biopsies are important methods to confirm bacteriological-negative tuberculosis. Endobronchial ultrasonography with guide sheath (EBUS-GS) and electromagnetic navigation bronchoscopy (ENB) are two new endobronchial diagnostic techniques, the combination of which has greatly facilitated the diagnosis of peripheral pulmonary lesions and is an especially useful, minimally invasive, effective diagnostic method for bacteriologically-negative tuberculosis cases.

Methods: A total of 78 patients were included in this study with suspected pulmonary tuberculosis based on clinical manifestations, laboratory tests, and imaging studies. The patients underwent a high-resolution chest CT scan before bronchoscopy, A method was selected (EBUS-GS alone, or EBUS-GS + ENB) based on the lesion site and the level and angle of the bronchus involved. After the lesion was found, a puncture needle, biopsy forceps, and brushing forceps were used to collect a tissue sample.

Results: Forty-four patients were diagnosed with tuberculosis; 1, nontuberculous Mycobacteria (NTM) lung disease; 15, lung cancer; 15, pulmonary infection; 1, allergic bronchopulmonary aspergillosis (ABPA); and 2, pneumoconiosis. A total of 25 patients of TB (56.8\%) were successfully diagnosed with EBUS-GS plus ENB. Among the patients with confirmed diagnosis, 9 were diagnosed with pathological examination; 4 , genetic analysis; 11, positive smear; and 14, positive culture.

Conclusions: The introduction of EBUS and ENB in China has provided a new direction for the diagnosis of atypical bacteriological-negative tuberculosis, as the techniques are less invasive and less expensive than thoracoscopy.

Keywords: Tuberculosis; diagnostics; endobronchial ultrasonography with guide sheath (EBUS-GS); electromagnetic navigation bronchoscopy (ENB)

Submitted Jun 07, 2019. Accepted for publication Aug 26, 2019.

doi: 10.21037/atm.2019.09.37

View this article at: http://dx.doi.org/10.21037/atm.2019.09.37 


\section{Introduction}

In the absence of a positive sputum bacteriological result, pathological and bacteriological examinations of lung lesion biopsies are important methods for confirming bacteriologically-negative tuberculosis (1). However, it is challenging to access the lesion in order to obtain a sufficient amount of tissue sample. Surgery is one of the most direct methods; however, it is invasive, expensive, and not suitable for all patient populations as a diagnostic technique. Percutaneous pulmonary puncture is a conventional method used for biopsy; it is minimally invasive and inexpensive and is thus widely used in clinical practice. However, this method is less precise when the lesion is tiny or locates at the inner one-third area of lung and is associated with high pneumothorax and bleeding rates (2). Tuberculosis specialists have thus been searching for an effective and minimally invasive method to access and diagnose lesions.

In endobronchial ultrasonography using guide sheath (EBUS-GS), a $360^{\circ}$ small ring-shaped ultrasound probe is used for transbronchial access of the peripheral pulmonary area. A guide sheath is then used to establish a channel for biopsy under the guidance of ultrasound imaging of the lesion (3). In electromagnetic navigation bronchoscopy (ENB), a flexible catheter is guided by real-time positioning to access peripheral pulmonary lesions for biopsy (4). These two new endobronchial diagnostic techniques, when combined, greatly facilitate the diagnosis of peripheral pulmonary lesions (5) and are an especially useful, minimally invasive, effective diagnostic method for difficult pulmonary tuberculosis cases.

EBUS and ENB were introduced into Shanghai Pulmonary Hospital, China, in 2010 and 2014, respectively, and Shanghai Pulmonary Hospital was the first hospital in the Asia Pacific region to adopt ENB (6). We have diagnosed 78 cases of suspected atypical bacteriologicallynegative pulmonary tuberculosis with these two techniques between June 2014 and March 2017. In this paper, we will discuss the clinical value of these two techniques.

\section{Methods}

\section{General information}

Among 126 cases from June 2014 to March 2017, $34(27 \%)$ cases showed lesions cannot reached by EBUS/ ENB indicated by chest CT, 7 (5.6\%) cases refused EBUS/ ENB, and 7 (5.6\%) cases lost to follow-ups. At last, a total of
78 patients were included in this prospective observational study, including 54 men and 24 women aged 19 to 76 (mean: $48 \pm 17$ ) years with suspected pulmonary tuberculosis based on clinical manifestations, laboratory tests, and imaging studies (Figure 1). Of these patients, 33 received 14 days to 24 months of diagnostic anti-tuberculosis treatment that did not confirm tuberculosis. Patients underwent high-resolution computed tomography (CT) before bronchoscopy (Figure 2), and data was recorded on a CD. In addition, patients underwent relevant preoperative tests and exams, including electrocardiograms (ECG), blood clotting time assessment, routine blood tests, and HIV, syphilis, and hepatitis marker screens, to ensure that they did not have any contraindications for bronchoscopy. The study was in accordance with the amended Declaration of Helsinki and approved by the Ethical Committee of Shanghai Pulmonary Hospital. Patients and their families were informed about the study and signed informed consent forms.

\section{Inclusion criteria}

Inclusion criteria were as follows: (I) Negative sputum acid-fast bacilli smear and negative sputum molecular test (repeated three times); (II) pulmonary tuberculosis diagnosed at another hospital with no marked absorption of the lesion after at least two months of diagnostic antituberculosis treatment; (III) unconventional lesion site and no signs of typical tuberculosis revealed by imaging studies; (IV) A method was selected (EBUS-GS alone, or EBUSGS + ENB) based on the lesion site and the level and angle of the bronchus involved; and 5) Difficult cases cannot diagnosed through regular bronchoscopy or any other examinations showed.

\section{Exclusion criteria}

Exclusion criteria were as follows: (I) patients cannot receive bronchoscopy due to contraindications; (II) diagnosed patients with confirmed acid-fast bacilli in sputum; and (III) patients' lesions $<1 \mathrm{~cm}$, without bronchus sign, or cannot reached by ENB.

\section{Preoperative preparation and anesthesia}

Patients were instructed to fast (no food or water) for at least six hours before bronchoscopy. Patients with hypertension were instructed to take hypotensive drugs, and patients with diabetes were instructed to skip a dose 


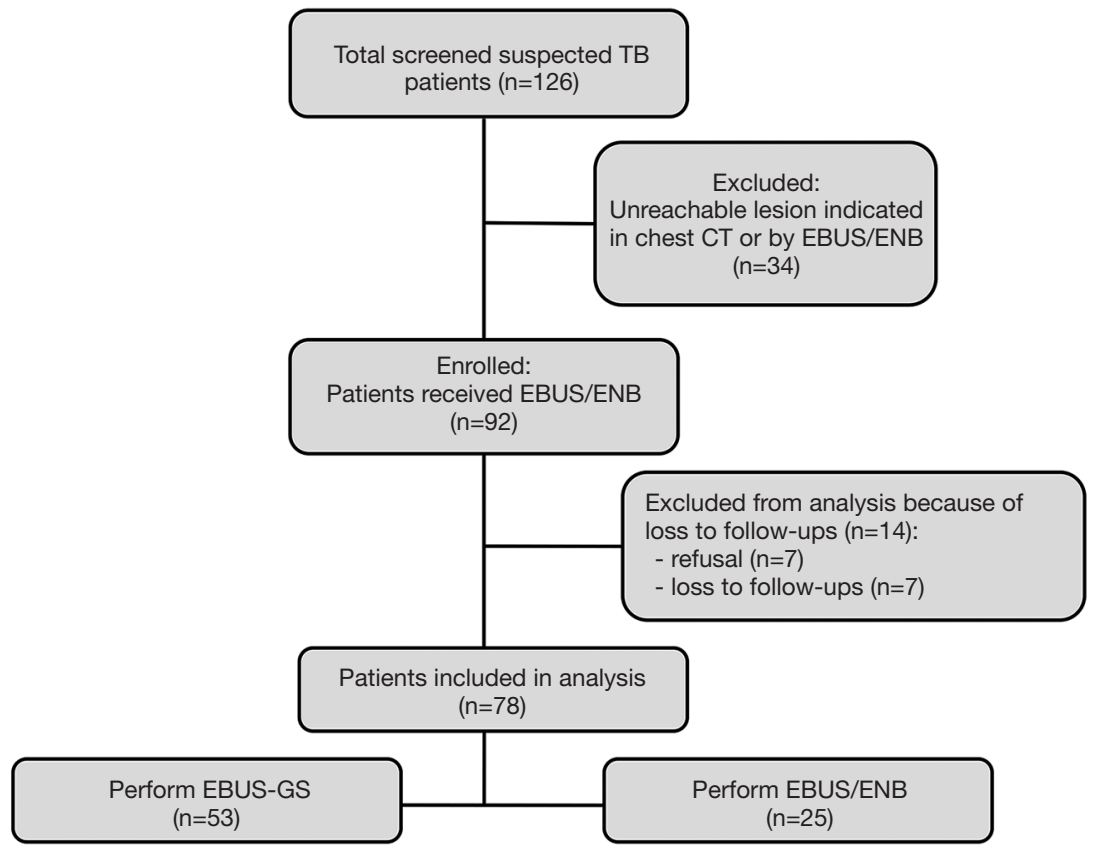

Figure 1 Flow diagram of total enrolled suspected tuberculosis patients from June 2014 to March 2017.

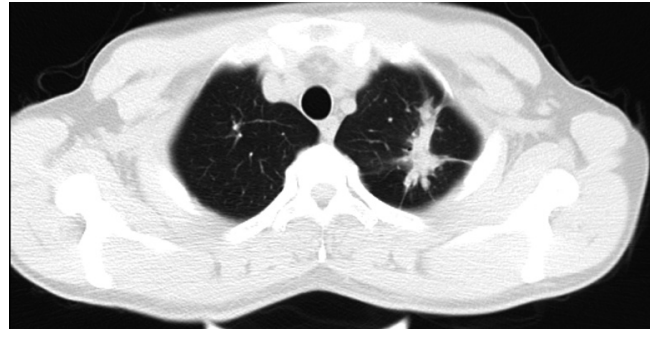

Figure 2 Chest CT scan of the patient showed the location and morphology of the lesion.

of hypoglycemic drugs or insulin. The bronchoscopy was performed with general anesthesia. Once in the operating theater, patients were placed in the supine position on the table. A peripheral venous access was established, and ECG, pulse oxygen saturation, and blood pressure were monitored. Sufentanil $(0.4-0.6 \mu \mathrm{g} / \mathrm{kg})$, propofol $(1.5-2 \mathrm{mg} / \mathrm{kg})$, and succinylcholine $(1-1.5 \mathrm{mg} / \mathrm{kg})$ were intravenously injected to induce general anesthesia. After $1 \mathrm{~min}$, a laryngeal mask was placed on the patient (male: $5^{\#}$, female: $4^{\#}$ ). During navigation, mechanical ventilation was administered, and anesthesia was maintained with propofol $(25-75 \mu \mathrm{g} / \mathrm{kg} / \mathrm{min})$ and succinylcholine $(3-5 \mu \mathrm{g} / \mathrm{kg} / \mathrm{min})$. At the end of bronchoscopy, naloxone $(0.2-0.4 \mathrm{mg})$ was administered, and the laryngeal mask was removed once the patient was completely awake.

\section{Equipment}

EBUS utilizes a CLV-260SL Xenon light source (Olympus Corporation, Japan), a CLV-260SL image processing station (Olympus Corporation, Japan), a BF-1T260 flexible bronchoscope (Olympus Corporation, Japan), an EU-ME1 endoscopic ultrasound system for peripheral ultrasonography (Olympus Corporation, Japan), and a UM-S30-20R endobronchial ultrasound probe (Olympus Corporation, Japan).

ENB (American SuperDimension, Ltd., USA) comprises five components: (I) a laptop with the inReach 6.1.3 system; (II) an electromagnetic positioning plate; (III) an electromagnetic catheter; (IV) an access tube; (V) an electromagnetic navigator.

\section{Examination methods}

(I) The patients underwent a high-resolution chest CT scan before bronchoscopy, and imaging data was recorded on a CD. A laptop with inReach software was used for bronchus reconstruction, registration, and access planning (7);

(II) A method was selected (EBUS-GS alone, or EBUS- 


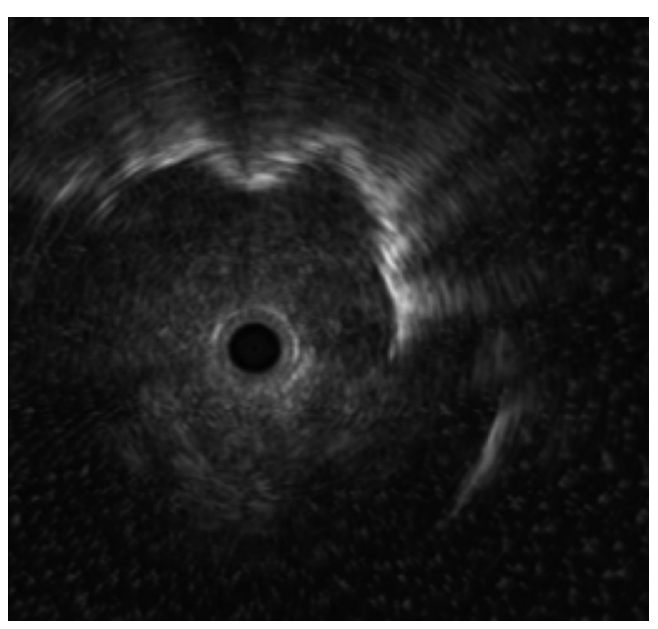

Figure 3 Ultrasound image of EBUS confirming the lesion site. EBUS, endobronchial ultrasonography.

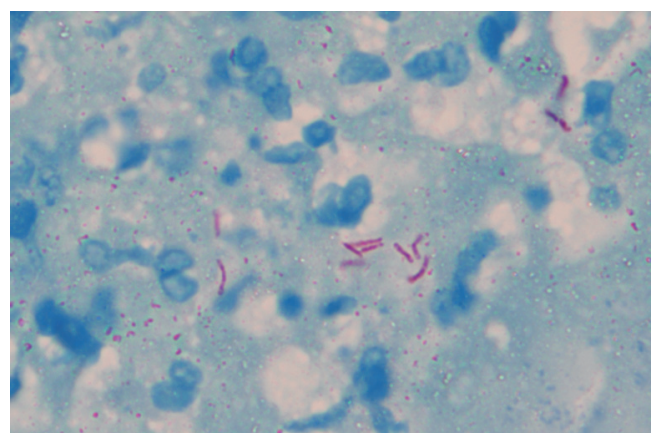

Figure 4 Pathological examination shows granuloma with cheesy necrosis, positive acid-fast staining, $\times 100$.

GS + ENB) based on the lesion site and the level and angle of the bronchus involved;

(III) For EBUS-GS alone, an ultrasound probe was guided by a bronchoscope to access the lesion via the planned path, and the lesion site was confirmed with ultrasound imaging;

(IV) For EBUS-GS + ENB, a catheter was first inserted and attached to the access channel, which was inserted into the trachea with bronchoscope clamps. The sensors at the front of the catheter were then used for registration (8). Once registration was completed, the bronchoscope was placed at the appropriate lesion segment, and the catheter was advanced to the lesion site under real-time navigation. After the channel was secured, the catheter was withdrawn, and the ultrasound probe was used to reconfirm the lesion site

\section{(Figure 3);}

(V) After the ultrasound probe was withdrawn, a puncture needle, biopsy forceps, and brushing forceps were used to collect a tissue sample via the channel and under fluoroscopy. Three valid samples were obtained by the forceps biopsy per patient. Saline $(5 \mathrm{~mL})$ was used to irrigate the channel, and the irrigation fluid was collected.

\section{Pathological and bacteriological examination, genetic sequencing and final diagnosis}

All brushing, needle puncture and aspiration, and irrigation fluid samples were sent to the clinical laboratory for acidfast bacilli smear, acid-fast bacilli solid culture, Bactec 960 MGIT culture, and liquid-based cytology. Each biopsy sample was fixed in formalin, embedded in paraffin, and stained with H\&E for histopathological examination, immunohistochemistry, and genetic analysis. Gold standards for diagnosis included granuloma with cheesy necrosis (histopathological examination), Mycobacterium tuberculosis (M. $t b)$ DNA segment detected in genetic sequencing of tissues, positive acid-fast staining or positive acid-fast bacilli smear, and positive M. $t b$ culture (Figure 4). Unconfirmed cases were subject to surgery or followed up for 12 months to confirm the diagnosis.

\section{Statistical analysis}

SPSS v19.0 was used for statistical analysis of data. Measurement data are expressed as $\bar{x} \pm$ SD. Count data are expressed as rates (\%).

\section{Results}

We examined a total of 78 patients with suspected pulmonary tuberculosis between June 2014 and March 2017 , including 54 men and 24 women with an average age of $48 \pm 17$ years. General information on the study population is presented in Table 1. Maximum diameter of the lesion is $29.1 \pm 14.5 \mathrm{~mm}$. Laboratory tests and previous treatments are presented in Table 2. Finally, 44 patients were diagnosed with tuberculosis, 1 with nontuberculous Mycobacterial (NTM) lung disease, 15 with lung cancer, 15 with pulmonary infection, 1 with allergic bronchopulmonary aspergillosis (ABPA), and 2 with pneumoconiosis. A total of 25 tuberculosis patients (56.8\%) were successfully diagnosed using EBUS-GS + ENB (Table 3). 
Table 1 General information and lesion characteristics

\begin{tabular}{lc}
\hline Characteristics & $\mathrm{n}(\%)$ \\
\hline Sex & $54(69.2)$ \\
Male & $24(30.8)$ \\
Female & \\
Symptoms & $46(59.0)$ \\
Cough & $14(17.9)$ \\
Coughing up blood & $13(16.7)$ \\
Low-grade fever & $24(30.8)$ \\
Found during checkup & \\
Previous medical history & $8(10.3)$ \\
Diabetes & $5(6.4)$ \\
COPD & $3(3.8)$ \\
Asthma & $2(2.6)$ \\
Bronchiectasis & \\
Course of disease & $39(50.0)$ \\
<3 months & $16(20.5)$ \\
3-6 months & $22(29.5)$ \\
\hline Cesion site & $28(35.9)$ \\
\hline
\end{tabular}

COPD, chronic obstructive pulmonary disease.

Among 78 cases, 53 cases received EBUS-GS, and then 36 cases were confirmed indicating a diagnostic rate of $67.9 \%, 25$ cases received EBUS-GS combining with $\mathrm{ENB}$, and 19 were diagnosed indicating a diagnostic rate of $76 \%$. Nine of the patients with confirmed diagnoses were diagnosed by pathological examination, 4 by genetic analysis, 11 by positive smears, and 14 by positive cultures. Among all confirmed tuberculosis patients, positive rate of brushing forceps was $20.5 \%$ (9/44), biopsy $45.5 \%$ (20/44), lavage fluid smear and culture $36.4 \%$ (16/44), and transbronchial needle aspiration $40 \%(4 / 10)$. No
Table 2 Preoperative laboratory tests and previous treatment regimens

\begin{tabular}{|c|c|}
\hline Laboratory tests and treatment regimens & $\mathrm{n}(\%)$ \\
\hline \multicolumn{2}{|l|}{ Tuberculosis antibody } \\
\hline Positive & $29(37.2)$ \\
\hline Negative & $41(52.6)$ \\
\hline Unknown & $8(10.3)$ \\
\hline \multicolumn{2}{|l|}{ T-SPOT } \\
\hline Positive & $36(46.2)$ \\
\hline Negative & $33(42.3)$ \\
\hline Unknown & $9(11.5)$ \\
\hline \multicolumn{2}{|l|}{ CRP } \\
\hline$<10$ & $54(69.2)$ \\
\hline$>10$ & $18(23.1)$ \\
\hline Unknown & $6(7.7)$ \\
\hline \multicolumn{2}{|l|}{ ESR } \\
\hline Normal & $43(55.1)$ \\
\hline Abnormal & $29(37.2)$ \\
\hline Unknown & $6(7.7)$ \\
\hline \multicolumn{2}{|l|}{ Conventional bronchoscopy } \\
\hline Positive & $0(0.0)$ \\
\hline Negative & $78(100.0)$ \\
\hline \multicolumn{2}{|l|}{ Pulmonary puncture } \\
\hline Inflammatory cells and epithelial cells & $19(24.4)$ \\
\hline \multicolumn{2}{|l|}{ Diagnostic anti-tuberculosis treatment } \\
\hline$<3$ months & $9(11.5)$ \\
\hline $3-6$ months & $5(6.4)$ \\
\hline$>6$ months & $19(24.4)$ \\
\hline
\end{tabular}

CRP, C-reactive protein; ESR, erythrocyte sedimentation rate.

complications such as pneumothorax, major bleeding, or infection were observed in this study.

\section{Discussion}

\section{Clinical application of the endobronchial technique}

Historically, respiratory specialists have relied on transbronchial lung biopsy (TBLB) to diagnose peripheral 
Table 3 Comparison of EBUS + ENB diagnosis results versus final diagnosis

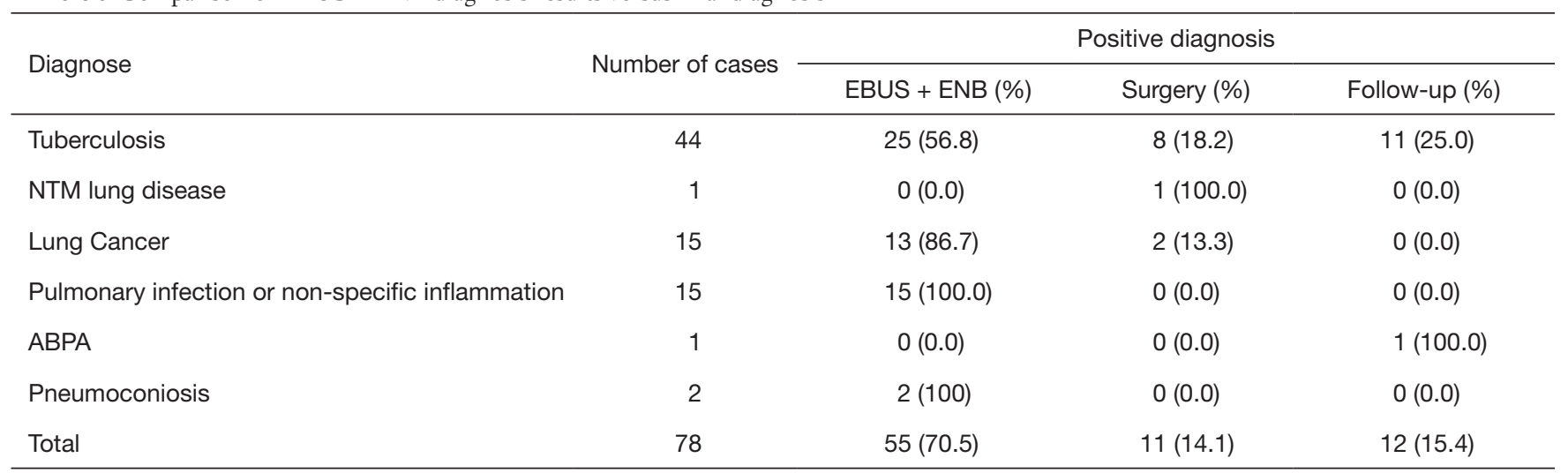

EBUS, endobronchial ultrasonography; ENB, electromagnetic navigation bronchoscopy; NTM, nontuberculous Mycobacteria; ABPA, allergic bronchopulmonary aspergillosis.

lung lesions, especially diffuse lesions. Given the increasing number of patients with peripheral pulmonary nodules in China, physicians have attempted lung aspiration or biopsy under the guidance of fluoroscopy, but the diagnostic rate is low (9). EBUS-GS, introduced into China in 2010, uses a ring-shaped ultrasound probe that can access bronchioles via bronchoscope clamps to observe the bronchus with ultrasound, locate the lesion, and collect a sample through the guide sheath. This technique is especially advantageous for peripheral lesions near the mediastinum (10). The technique is performed through natural channels, thereby minimizing damage and pneumothorax. Ultrasound images confirm the location of vessels around the lesion, which helps prevent major bleeding (11).

EBUS-GS cannot access distal bronchi and is inflexible. As a result, it cannot locate certain distal lesions or lesions with sharp bronchial angles. But, since first introduced into clinical practice in the US in 2005, ENB has contributed to major breakthroughs in locating and diagnosing small peripheral pulmonary nodules (12). The SuperDimension inReach system uses the ENB technique for minimally invasive exploration of deep lung lesions and mediastinal lymph nodes. The system has sensors at the front of the catheter that send signals to three magnetic nodes on the patient's chest and the electromagnetic positioning plate under the patient's body, thus acting like a GPS navigation system in the patient's lungs. The system guides the biopsy device to suspected sites to diagnose suspected target tissue.

ENB has a high diagnostic rate for both benign and malignant peripheral pulmonary nodules (13). When used with EBUS, ENB can confirm the lesion in real time, further improving the diagnostic rate (14), minimizing bleeding, and reducing patient and operator exposure to $\mathrm{X}$-rays. Thus, this combination represents a minimally invasive, effective, and safe endobronchial diagnostic technique.

\section{Application of the endobronchial technique in the diagnosis of difficult tuberculosis cases}

It has been difficult to diagnose and treat atypical bacteriological-negative pulmonary tuberculosis because of the lack of "gold standards" for diagnosis. Such cases are typically diagnosed based on imaging studies, tuberculosis antibodies, and the T-SPOT assay (15). Invasive exams, such as bronchoscopy and percutaneous pulmonary puncture, may improve the detection rate of Mycobacterium tuberculosis (16-18). In recent years, interferon- $\gamma$ release assays and rapid molecular detection assays have greatly advanced the diagnosis of pulmonary tuberculosis. Diagnostic anti-tuberculosis treatment also plays an important role in the diagnosis and treatment of bacteriologically-negative tuberculosis and is thus widely used in clinical practice. However, It is important that histopathological examination is routinely introduced to confirm tuberculosis in unconfirmed cases using EBUSGS or ENB, especially in patients who do not respond to diagnostic anti-tuberculosis treatment.

At present, most foreign clinical studies on peripheral endobronchial techniques focus on malignant diseases and have demonstrated the effectiveness of EBUS and ENB use for the diagnosis of malignant nodules. Our previous case report demonstrated that ENB has specific clinical value in the diagnosis of bacteriologically-negative pulmonary 
tuberculosis (19). In this study, all 78 patients were suspected to have pulmonary tuberculosis on the basis of symptoms, laboratory tests, and chest imaging studies. Of these, 33 patients received 14 days to 24 months of diagnostic anti-tuberculosis treatment, and repeated sputum acid-fast bacilli smears and cultures were negative. Conventional bronchoscopy and pulmonary puncture, however, could not confirm the diagnosis. In $71.8 \%$ of the patients, the lesion was located in the posterior, apical, or dorsal segment of the upper lobes, was inaccessible by conventional bronchoscopy, and a second percutaneous pulmonary puncture would have been risky or difficult to perform and had a low positive rate. When using EBUS plus ENB (brushing, biopsy, irrigation), however, $70.5 \%$ of the cases were pathologically or bacteriologically confirmed, and complications such as pneumothorax did not occur. Our results demonstrate that EBUS plus ENB is advantageous for enabling lesion access and successful biopsy and diagnosis with various detection methods. Among patients with a final diagnosis of pulmonary tuberculosis, $56.8 \%$ were confirmed with EBUS plus ENB. The diagnostic rate of this combined technique was relatively low for malignant lesions but enabled a breakthrough for benign lesions, indicating that EBUS plus ENB is a promising technique for the differential diagnosis of atypical bacteriological-negative tuberculosis.

\section{Advantage and dis advantage of the endobronchial technique in tuberculosis diagnosis}

Studies have demonstrated the feasibility of electromagnetic navigation and EBUS (20). With constant improvements and upgrades, the electromagnetic navigation technique is expected to facilitate the identification of even smaller lesions and be more widely used in the future. The cost of EBUS-GS/ENB is $15000 \mathrm{CNY}$ in China, which is more expensive than regular bronchoscopy (400 CNY) together with laboratory test $(1,000 \mathrm{CNY})$, while the cost of thoracoscopy is about 40,000 CNY. In this study, the diagnostic rate of EBUS-GS/ENB was $75 \%$, while the diagnostic rate of thoracoscopy was $100 \%$ (21). Though the diagnostic rate of thoracoscopy is high, it has high cost, big invasiveness, and many complications. EBUS-GS/ ENB is a method worthy recommendation. However, these techniques are not yet mature and require further validation and investigation to confirm their clinical value in the diagnosis and treatment of tuberculosis.

There are some limitations in this study. This is an observational study, describing the application of EBUS-
GS/ENB in culture negative tuberculosis patients. However, there was no control group, and the method was not compared with other methods. In the future, comparison will be conducted between EBUS-GS/ENB and other methods such as thoracoscopy and traditional diagnostic methods in terms of diagnostic value and costeffective, aiming to reveal the application value of EBUSGS/ENB in culture negative tuberculosis patients more deeply and comprehensively. Due to the lack of relative international guidelines and publications, the choice of EBUS-GS/ENB is relatively subjective. There is still a lack of unified and specific standard. In the future, detailed indications of EBUS-GS and BUS-GS/ENB can be concluded through large sample studied, to make BUS-GS/ ENB more reasonable and more effectively used.

In conclusion, the introduction of EBUS and ENB in China has provided a new direction for the diagnosis of atypical bacteriological-negative tuberculosis, as the techniques are less invasive and less expensive than thoracoscopy.

\section{Acknowledgments}

Funding: This study was supported by the Shanghai Pujiang Program (16PJD041) and Clinical Research Plan of SHDC (16CR1028B).

\section{Footnote}

Conflicts of Interest: The authors have no conflicts of interest to declare.

Etbical Statement: The authors are accountable for all aspects of the work in ensuring that questions related to the accuracy or integrity of any part of the work are appropriately investigated and resolved. The study was in accordance with the amended Declaration of Helsinki and approved by the Ethical Committee of Shanghai Pulmonary Hospital (18Q016NJ). Patients and their families were informed about the study and signed informed consent forms.

\section{References}

1. Dhamija A, Ganga VB, Guliani A, et al. Endobronchial ultrasound for tubercular mediastinal adenopathy and its comparison with traditional tools. Int J Tuberc Lung Dis 2019;23:907-12.

2. Gould MK, Fletcher J, Iannettoni MD, et al. Evaluation of 
patients with pulmonary nodules: when is it lung cancer?: ACCP evidence-based clinical practice guidelines (2nd edition). Chest 2007;132:108S-30S.

3. Paone G, Nicastri E, Lucantoni G, et al. Endobronchial ultrasound-driven biopsy in the diagnosis of peripheral lung lesions. Chest 2005;128:3551-7.

4. Mahajan AK, Patel S, Hogarth DK, et al. Electromagnetic navigational bronchoscopy: an effective and safe approach to diagnose peripheral lung lesions unreachable by conventional bronchoscopy in high-risk patients. J Bronchology Interv Pulmonol 2011;18:133-7.

5. Krimsky W, Seijo LM. Bronchoscopy and the peripheral nodule in the age of lung cancer screening and targeted therapies. Curr Respir Care Rep 2012;1:67-71.

6. Gu Y, Chen S, Shi J, et al. The introduction of electromagnetic navigation bronchoscopy for the diagnosis of small pulmonary peripheral lesions in an Asian population. J Thorac Dis 2017;9:2959-65.

7. Seijo LM, de Torres JP, Lozano MD, et al. Diagnostic yield of electromagnetic navigation bronchoscopy is highly dependent on the presence of a Bronchus sign on CT imaging: results from a prospective study. Chest 2010;138:1316-21.

8. Pearlstein DP, Quinn CC, Burtis CC, et al. Electromagnetic navigation bronchoscopy performed by thoracic surgeons: one center's early success. Ann Thorac Surg 2012;93:944-9; discussion 949-50.

9. Rivera MP, Mehta AC, American College of Chest Physicians.. Initial diagnosis of lung cancer: ACCP evidence-based clinical practice guidelines (2nd edition). Chest 2007;132:131S-48S.

10. Kurimoto N, Miyazawa T, Okimasa S, et al. Endobronchial ultrasonography using a guide sheath increases the ability to diagnose peripheral pulmonary lesions endoscopically. Chest 2004;126:959-65.

Cite this article as: $\mathrm{Gu} \mathrm{Y,} \mathrm{Wu} \mathrm{C,} \mathrm{Yu} \mathrm{F,} \mathrm{Gui} \mathrm{X,} \mathrm{Ma} \mathrm{J,} \mathrm{Cheng} \mathrm{L,}$ Sun Q, Sha W. Application of endobronchial ultrasonography using a guide sheath and electromagnetic navigation bronchoscopy in the diagnosis of atypical bacteriologicallynegative pulmonary tuberculosis. Ann Transl Med 2019;7(20):567. doi: $10.21037 /$ atm.2019.09.37
11. Kurimoto N, Murayama M, Yoshioka S, et al. Analysis of the internal structure of peripheral pulmonary lesions using endobronchial ultrasonography. Chest 2002;122:1887-94.

12. Leong S, Ju H, Marshall H, et al. Electromagnetic navigation bronchoscopy: A descriptive analysis. J Thorac Dis 2012;4:173-85.

13. Gildea TR, Mazzone PJ, Karnak D, et al. Electromagnetic navigation diagnostic bronchoscopy: a prospective study. Am J Respir Crit Care Med 2006;174:982-9.

14. Chee A, Stather DR, Maceachern P, et al. Diagnostic utility of peripheral endobronchial ultrasound with electromagnetic navigation bronchoscopy in peripheral lung nodules. Respirology 2013;18:784-9.

15. Chinese Medical Association Tuberculosis Branch. Guidelines for the diagnosis and treatment of tuberculosis. Chin J Tuberc Respir Dis 2001;24:70-4.

16. Sun J, Teng J, Yang H, et al. Endobronchial ultrasound-guided transbronchial needle aspiration in diagnosing intrathoracic tuberculosis. Ann Thorac Surg 2013;96:2021-7.

17. Soto A, Acurio V, Solari L, et al. Incremental yield of bronchial washing for diagnosing smear-negative pulmonary tuberculosis. Rev Saude Publica 2013;47:813-6.

18. Yoo H, Song JU, Koh WJ, et al. Additional role of second washing specimen obtained during single bronchoscopy session in diagnosis of pulmonary tuberculosis. BMC Infect Dis 2013;13:404.

19. Port J, Harrison S. Electromagnetic navigational bronchoscopy. Semin Intervent Radiol 2013;30:128-32.

20. Bechara R, Parks C, Ernst A. Electromagnetic navigation bronchoscopy. Future Oncol 2011;7:31-6.

21. Hsu KY, Lee HC, Ou CC, et al. Value of video-assisted thoracoscopic surgery in the diagnosis and treatment of pulmonary tuberculoma: 53 cases analysis and review of literature. J Zhejiang Univ Sci B 2009;10:375-9. 